\title{
Epiphora following chemotherapy with pemetrexed in patients with advanced non-small cell lung cancer
}

\author{
Yun Duk Jung ${ }^{1}$, Sang Bin Lee ${ }^{1}$, Yun Wha Jung ${ }^{1}$, Jung Sub Song ${ }^{2}$, and In Sook Woo
}

Divisions of ${ }^{1} \mathrm{Hematology}$ and Medical Oncology and ${ }^{2}$ Pulmonology, Department of Internal Medicine, College of Medicine, Yeouido St. Mary's Hospital, The Catholic University of Korea, Seoul, Korea

Received: February 18, 2015

Revised : March 25, 2015

Accepted: May 12, 2015

\section{Corresponding to}

In Sook Woo, M.D.

Division of Hematology and

Medical Oncology, Department of

Internal Medicine, College of

Medicine, Yeouido St. Mary's

Hospital, The Catholic University of

Korea, 10 63-ro, Yeongdeungpo-gu,

Seoul 07345, Korea

Tel: $+82-2-3779-1574$

Fax: +82-2-780-3132

E-mail: insookwoo@catholic.ac.kr
To the Editor,

Anticancer drugs may damage the epithelial cells of the conjunctival mucosa. Inflammation and fibrosis of epithelial cells of the lacrimal duct following systemic chemotherapy may lead to lacrimal outflow obstruction and epiphora. Excessive lacrimation could be underestimated as a minor problem by both physicians and patients with cancer who undergo chemotherapy. However, epiphora may have a negative impact on the quality of life of patients and infection can be caused by obstruction of the lacrimal duct. Anticancer chemotherapeutic drugs causing epiphora include oxaliplatin, cyclophosphamide, 5-fluorouracil (5-FU), docetaxel, doxorubicin, cytosine arabinoside, pentostatin, and panitumumab [1]. Pemetrexed is a multi-target anti-folate metabolite that acts by inhibiting thymidylate synthase, dihydrofolate reductase, and glycinamide ribonucleotide formyltransferase, all of which are folate dependent enzymes involved in the de novo biosynthesis of thymidine and purine nucleotides. It is used to treat malignant pleural mesothelioma and is administered for a prolonged period as maintenance therapy until disease progression for non-squamous cell type of advanced lung cancer. The toxicities associated with pemetrexed include gastroin- testinal upsets (nausea, diarrhea, and vomiting), bone marrow suppression, asthenia, and rashes [2]. In terms of the ocular problem with pemetrexed, conjunctivitis and periorbital edema occur in $25 \%$ and $15 \%$ of 107 patients administered with four cycles or more of pemetrexed, respectively [1]. pemetrexed may also cause excessive lacrimation. To the best of our knowledge, excessive tearing associated with pemetrexed has never been reported in medical literature although pemetrexed has been clinically applied for 10 years. A 63-year-old male patient was referred to our department owing to the detection of pulmonary nodules on chest computed tomography (CT) performed in another hospital. The patient was a never-smoker and had been previously diagnosed with hypertension, and was receiving medication at time. Chest CT revealed one nodule that was $1.7 \mathrm{~cm}$ in size with an irregular shape in the anterior segment of the left upper lobe of the lung, multifocal small nodular ground glass opacity on both lungs, and two nodules in the right upper lobe of the lung. Adenocarcinoma with bronchioalveolar carcinoma was diagnosed via a percutaneous needle aspiration biopsy of the left upper lobe nodule and wedge resection of the anterior segment of the left upper lobe. Samples were determined 
to be negative for mutation of epidermal growth factor receptor and amplication of anaplastic lymphoma kinase via fluorescence in situ hybridization. Distant metastasis was not observed with the exception of lung to lung metastasis.

Seven months later, the patient's chest CT showed an increase in the solid portion of multifocal nodules of right upper lobe of the lung. Combination chemotherapy involving gemcitabine and cisplatin was administered as first line therapy. After six cycles, the patient was considered to have stable disease. Two options were suggested to the patient: to alternate to maintenance therapy with pemetrexed, or to undergo observation. The patient chose observation. After 2 months, his chest CT showed an increment in the size of nodules in right upper lobe. Pemetrexed monotherapy $\left(500 \mathrm{mg} / \mathrm{m}^{2}\right)$ was administered every 3 weeks. Adverse events were not observed, with the exception of fatigue that was experienced during the treatment with pemetrexed. However, after four cycles of pemetrexed, the patient presented with excessive lacrimation that did not improve with conservative treatments such as artificial tears and xerophthalmia was suspected. Subsequent to consultation with an ophthalmologist, the patient was diagnosed with lacrimal punctal and canaliculi stenosis. His epiphora was resolved after surgery for punctal stenosis (3-snip punctoplasty) and canaliculi stenosis (probing and irrigation) followed by 4 weeks of topical corticosteroid therapy. The patient refused further chemotherapy. Regular follow-up is being performed at the outpatient clinic. The patient has not complained of epiphora since.

According to research concerning the ophthalmologic complications surrounding other anticancer agents (including docetaxel and 5-FU), ocular complications such as epiphora and conjunctivitis have been reported [1]. Lacrimation after treatment with docetaxel might be due to canaliculi and nasolacrimal duct obstruction. It has been histologically discovered that docetaxel caused chronic inflammation and fibrosis in the lacrimal sac when it was secreted through tears [3]. Similarly, squamous metaplasia with lumen narrowing was observed in histopathologic examinations of surgical specimens from patients administered 5-FU. According to research conducted on patients administered with 5 -FU, lacrimation was hypothesized to be due to increased reflux (tearing) from anatomic obstruction and secondary irritation of the cornea and conjunctiva. Lacrimation was also caused by hypersensitivity reactions to oxaliplatin [4]. The mechanism may involve the formation of immunogenic conjugates due to type 1 hypersensitivity reactions between cellular proteins and drug metabolites. Desensitization treatments such as dexamethasone, diphenhydramine, and intravenous fluid are effective in such patients [4]. According to a study by Eguia et al. [5] regarding the relationship between prolonged pemetrexed treatment and cutaneous adverse events, conjunctivitis can occur as an early marker of cutaneous adverse events, such as inflammatory edema. However, lacrimation was not mentioned in the study. The mechanism of lacrimation caused by treatment with pemetrexed might be due to the hindrance of tear flow from lacrimal duct stenosis and fibrosis of punctum, which also occurs following treatment with docetaxel or 5-FU. To diagnose epiphora associated with chemotherapy in patients, other causes of excessive lacrimation should be excluded.

In conclusion, epiphora associated with the chemotherapy involving pemetrexed may damage the quality of life of patients. Attention to the adverse effects following treatment with pemetrexed, and early consultation with an ophthalmologist is warranted to prevent reduction in quality of life of patients with cancer.

Keywords: Lacrimal apparatus diseases; Pemetrexed; Lung neoplasms

\section{Conflict of interest}

No potential conflict of interest relevant to this article was reported.

\section{REFERENCES}

1. Hazin R, Abuzetun JY, Daoud YJ, Abu-Khalaf MM. Ocular complications of cancer therapy: a primer for the ophthalmologist treating cancer patients. Curr Opin Ophthalmol 2009;20:308-317.

2. Hazarika M, White RM, Johnson JR, Pazdur R. FDA drug approval summaries: pemetrexed (Alimta). Oncologist 2004;9:482-488.

3. Esmaeli B, Burnstine MA, Ahmadi MA, Prieto VG. Docetaxel-induced histologic changes in the lacrimal sac and the nasal mucosa. Ophthal Plast Reconstr Surg 
2003;19:305-308.

4. Gowda A, Goel R, Berdzik J, Leichman CG, Javle M. Hypersensitivity reactions to oxaliplatin: incidence and management. Oncology (Williston Park) 2004;18:1671-
1675.

5. Eguia B, Ruppert AM, Fillon J, et al. Skin toxicities compromise prolonged pemetrexed treatment. J Thorac Oncol 2011;6:2083-2089. 